\title{
Approximation by $T$-Transformation of Double Walsh-Fourier Series to Multivariable Functions
}

\author{
Yi Zhao' and Dansheng Yu' \\ ${ }^{1}$ Department of Mathematics, Hangzhou Dianzi University, Hangzhou, Zhejiang 310018, China \\ ${ }^{2}$ Department of Mathematics, Hangzhou Normal University, Hangzhou, Zhejiang 310036, China \\ Correspondence should be addressed to Yi Zhao; mathyizhao@gmail.com
}

Received 20 January 2014; Accepted 9 March 2014; Published 22 April 2014

Academic Editors: D. G. Costa, C. Gutiérrez, and D.-X. Zhou

Copyright (C) 2014 Y. Zhao and D. Yu. This is an open access article distributed under the Creative Commons Attribution License, which permits unrestricted use, distribution, and reproduction in any medium, provided the original work is properly cited.

We study the Walsh series expansion of multivariate functions in $L_{p}(1 \leq p \leq \infty)$ and, in particular, in $\operatorname{Lip}(\alpha, p)$. The rate of uniform approximation by $T$-transformation of rectangular partial sums of double Walsh to these functions is investigated. By extending the concepts of rest (head) bounded variation series, which was introduced by Leindler (2004), we generalize the related results of Móricz and Rhoades (1996), Nagy (2012). Our results can be applied to many summability methods, including the Nörlund summability and weighted summability.

\section{Introduction}

Let $r_{0}(x)$ be the function defined on $I:=I_{0}=[0,1)$ by

$$
r_{0}(x)=\left\{\begin{array}{ll}
1, & x \in\left[0, \frac{1}{2}\right), \\
-1, & x \in\left[\frac{1}{2}, 1\right)
\end{array} r_{0}(x+1)=r_{0}(x) .\right.
$$

The Rademacher system is defined by

$$
r_{n}(x)=r_{0}\left(2^{n} x\right) \text {. }
$$

Let

$$
w_{0}(x)=1, \quad w_{n}(x)=\prod_{k=0}^{\infty}\left(r_{k}(x)\right)^{n_{k}}
$$

be the Walsh functions, where $x \in[0,1), n \in \mathbb{N}$ with the dyadic expansion $x=\sum_{i=0}^{\infty} x_{i} 2^{-i-1}$ and $n=\sum_{j=0}^{\infty} n_{j} 2^{j}$, respectively; here $x_{i}, n_{j} \in\{0,1\}$ for $i, j=0,1, \ldots$.. We also write $\lceil n\rceil:=\left\{\max j, n_{j} \neq 0\right\}$. The idea of using products of Rademacher's functions to construct the Walsh system originated from Paley [1].

As an important orthonormal bases, Walsh functions have most of the properties of Fourier series but are more suited to nonlinear studies. If a zero-memory nonlinear transformation is applied to a Walsh series, the output series can be derived by simple algebraic processes. Corrington [2] proved that nonlinear differential and integral equations can be solved by Walsh series. Meanwhile, the Walsh functions are of great practical interest. They have many applications in signal processing [3], dynamic systems, identification, control $[4,5]$, and so on.

In the above-mentioned issues, Walsh series expansion of certain function and its convergence to that function play very important roles. In this paper, we are interested in the Walsh expansion of multivariate functions and discuss the convergence of its $T$-transformation to these functions (we mention here that, in order to avoid notational difficulties, we restrict ourselves to the case of bivariate functions). Furthermore, the results are applied to some summability methods.

The Walsh-Dirichlet kernels and Walsh-Fejér kernels are defined by

$$
\begin{gathered}
D_{n}(x)=\sum_{k=0}^{n-1} w_{k}(x), \quad D_{0}(x):=0, \\
K_{n}(x)=\frac{1}{n} \sum_{k=1}^{n} D_{k}(x), \quad(n \in \mathbb{N}), K_{0}(x)=0 .
\end{gathered}
$$


It is known that [6]

$$
D_{2^{n}}(x)= \begin{cases}2^{n}, & x \in I_{n} \\ 0, & x \in I \backslash I_{n},\end{cases}
$$

where $I_{n}$ denotes the dyadic interval in $[0,1)$ defined by $I_{n}=$ $\left[\left(i / 2^{n}\right),\left((i+1) / 2^{n}\right)\right]$, and $i=0,1, \ldots, 2^{n}-1, n \in \mathbb{N}$.

In addition, we point out that the standard representations for the Walsh-Dirichlet kernel $[7,8]$ are

$$
\begin{gathered}
D_{2^{n}+j}(x)=D_{2^{n}}(x)+r_{n}(x) D_{j}(x), \\
D_{2^{j}+i}(x)-D_{2^{j+1}}(x)=-w_{2^{j+1}-1}(x) D_{2^{j}-i}(x) .
\end{gathered}
$$
that

For the Walsh-Fejér kernel $K_{i}$, let $i \geq 1$; Yano [9] proved

$$
\left\|K_{i}\right\|_{1} \leq 2
$$

Let $T:=\left\{t_{m n j k}\right\}$ be a doubly infinite matrix. It is said to be doubly triangular if $t_{m n j k}=0$ for $j>m$ or $k>n$. In the recent research [10], the authors established necessary conditions for a general inclusion theorem involving a pair of doubly triangular matrices.

Given a double sequence $\left\{s_{j k}: j, k=0,1, \ldots\right\}$ of complex numbers, the $m n$th term of the $T$-transformation of $\left\{s_{j k}\right\}$ is defined by

$$
t_{m n}:=\sum_{\mu=0}^{m} \sum_{\nu=0}^{n} t_{m n \mu \nu} s_{\mu \nu}
$$

If $\sum_{\mu=0}^{m} \sum_{\nu=0}^{n} t_{m n \mu \nu}=1$, then we say that $T$ is normal.

Let $P=\left\{p_{j k}: j, k=0,1, \ldots\right\}$ be a double sequence of nonnegative numbers; $p_{00}>0$. Taking

$$
\begin{aligned}
& t_{m n j k}=\frac{p_{m-j, n-k}}{P_{m n}}, \quad 0 \leq j \leq m, 0 \leq k \leq n, \\
& t_{m n j k}=0, \quad \text { if } j>m \text { or } k>n,
\end{aligned}
$$

where $P_{m n}:=\sum_{j=0}^{m} \sum_{k=0}^{n} p_{j k}$. Then the corresponding $t_{m n}$ is known as the Nörlund means. The Cesàro summability of orders $\gamma, \delta>-1$, denoted by $(C, \gamma, \delta)$, is a special case of the Nörlund summability with

$$
\begin{gathered}
p_{j k}=A_{j}^{\gamma-1} A_{k}^{\delta-1}, \quad j, k=0,1,2, \ldots, \\
A_{l}^{\gamma}=\left(\begin{array}{c}
\gamma+l \\
l
\end{array}\right)=\frac{(\gamma+1)(\gamma+2) \cdots(\gamma+l)}{l !}
\end{gathered}
$$

for $l=1,2, \ldots$, and $A_{0}^{\gamma}=1$. In this case,

$$
P_{m n}=A_{m}^{\gamma} A_{n}^{\delta}, \quad m, n=0,1,2, \ldots
$$

If we take

$$
\begin{aligned}
& t_{m n j k}=\frac{p_{j k}}{P_{m n}}, \quad 0 \leq j \leq m, 0 \leq k \leq n, \\
& t_{m n j k}=0, \quad \text { if } j>m \text { or } k>n,
\end{aligned}
$$

the corresponding $t_{m n}$ is the well-known Riesz means of $\left\{s_{j k}\right\}$.

Let $L_{p}(1 \leq p \leq \infty)$ denote the Lebesgue function spaces on the torus $I^{2}$; that is, $f \in L_{p}\left(I^{2}\right)$. The double Walsh (WalshFourier) series of such function is defined by

$$
f(x, y) \sim S(x, y)=\sum_{i=0}^{\infty} \sum_{j=0}^{\infty} \widehat{f}(i, j) w_{i}(x) w_{j}(y),
$$

and the $u v t$ th rectangular partial sum of $S$ is

$$
S_{u v}(x, y)=\sum_{i=0}^{u-1} \sum_{j=0}^{v-1} \widehat{f}(i, j) w_{i}(x) w_{j}(y),
$$

where

$$
\widehat{f}(i, j)=\iint_{0}^{1} f(s, t) w_{i}(s) w_{j}(t) d s d t .
$$

The $m n$th $T$-transformation of $S_{u v}$ is defined by

$$
T_{m n}:=T_{m n}(f, x, y)=\sum_{u=0}^{m} \sum_{v=0}^{n} t_{m n u v} S_{u v}(x, y) .
$$

By (16), we have

$$
T_{m n}(f, x, y)=\iint_{0}^{1} f(x+s, y+t) K_{m n}(s, t) d s d t
$$

where

$$
K_{m n}(s, t):=\sum_{u=0}^{m} \sum_{v=0}^{n} t_{m n u v} D_{u}(s) D_{v}(t), \quad m, n=0,1, \ldots,
$$

$D_{u}(s)$ and $D_{v}(t)$ are the Walsh-Dirichlet kernels, in terms of $u$ and $v$ respectively.

For any function $f \in L_{p}\left(I^{2}\right)$, when $T$ is normal,

$$
\begin{aligned}
& T_{m n}(x, y)-f(x, y) \\
& =\iint_{0}^{1} \sum_{u=0}^{m} \sum_{v=0}^{n} t_{m n u v} D_{u}(s) D_{v}(t) \\
& \quad \times(f(x+s, y+t)-f(x, y)) d s d t \\
& =\iint_{0}^{1} K_{m n}(s, t)(f(x+s, y+t)-f(x, y)) d s d t .
\end{aligned}
$$

Recall that the modulus of continuity of the function $f(x, y) \in L_{p}\left(I_{2}\right)$, 1-periodic in each variable, is defined by

$$
\begin{gathered}
\omega^{p}(f, \delta):=\sup _{|t| \leq \delta}\left\{\|f(x+u, y+v)-f(x, y)\|_{p},\right. \\
\left.|t|=\sqrt{u^{2}+v^{2}}, \delta \geq 0\right\} .
\end{gathered}
$$

For each $\alpha>0$, the Lipschitz classes in $L_{p}$ are defined by

$$
\operatorname{Lip}(\alpha, p):=\left\{f \in L_{p}, \omega^{p}(f, \delta)=\mathcal{O}\left(\delta^{\alpha}\right)\right\} .
$$


The (total) modulus of continuity of function $f(x, y) \in$ $L_{p}\left(I_{2}\right)$, 1-periodic in each variable, is defined by

$$
\begin{gathered}
\omega_{1,2}^{p}\left(f, \delta_{1}, \delta_{2}\right):=\sup \{\| f(x+u, y+v)-f(x+u, y) \\
-f(x, y+v)+f(x, y) \|_{p}, \\
\left.|u| \leq \delta_{1},|v| \leq \delta_{2}\right\} .
\end{gathered}
$$

It is easy to verify that there is a constant $\mathscr{C}>0$ such that

$$
\omega_{1,2}^{p}\left(f, \delta_{1}, \delta_{2}\right) \leq \mathscr{C}\left(\omega^{p}\left(f, \delta_{1}\right)+\omega^{p}\left(f, \delta_{2}\right)\right) .
$$

Móricz and Siddiqi [11] studied the rate of uniform approximation by Nörlund means of Walsh (Walsh-Fourier) series of $f \in L_{p}[0,1)$. Later, Móricz and Rhoades [12] studied the corresponding approximation problem by weighted means of Walsh-Fourier series. Their main results in [12] can be read as follows.

Theorem A. Let $f \in L_{p}, 1 \leq p \leq \infty, n=2^{m}+k, 1 \leq k \leq$ $2^{m}, m \geq 1$.

(i) If $\left\{p_{k}\right\}$ is nondecreasing and satisfies the condition

$$
\frac{n p_{n}}{P_{n}}=\mathcal{O}(1)
$$

then

$$
\begin{aligned}
\| t_{n}(f) & -f \|_{p} \\
& \leq \frac{3}{P_{n}} \sum_{j=0}^{m-1} 2^{j} p_{2^{j+1}-1} \omega_{p}\left(f, 2^{-j}\right)+\mathcal{O}\left(\omega_{p}\left(f, 2^{-m}\right)\right) .
\end{aligned}
$$

(ii) If $\left\{p_{k}\right\}$ is nonincreasing, then

$$
\left\|t_{n}(f)-f\right\|_{p} \leq \frac{3}{P_{n}} \sum_{j=0}^{m-1} 2^{j} p_{2^{j}} \omega_{p}\left(f, 2^{-j}\right)+\mathcal{O}\left(\omega_{p}\left(f, 2^{-m}\right)\right) .
$$

Theorem B. Let $f \in \operatorname{Lip}(\alpha, p)$ for $\alpha>0$ and $1 \leq p \leq \infty$. If $\left\{p_{k}\right\}$ is nondecreasing, then one has the following estimates:

$$
\left\|t_{n}(f)-f\right\|_{L_{p}}= \begin{cases}\mathcal{O}\left(n^{-\alpha}\right), & 0<\alpha<1, \\ \mathcal{O}\left(n^{-1} \log n\right), & \alpha=1, \\ \mathcal{O}\left(n^{-1}\right), & \alpha>1 .\end{cases}
$$

For any fourfold sequence $\left\{a_{m n j k}\right\}$, write

$$
\begin{gathered}
\Delta_{11} a_{m n j k}:=a_{m n j k}-a_{m, n, j+1, k}-a_{m, n, j, k+1}+a_{m, n, j+1, k+1}, \\
\Delta_{01} a_{m n j k}:=a_{m n j k}-a_{m, n, j, k+1}, \\
\Delta_{10} a_{m n j k}:=a_{m n j k}-a_{m, n, j+1, k} .
\end{gathered}
$$

The sequence $\left\{a_{m n j k}\right\}$ is called nondecreasing if it is nondecreasing in both $j$ and $k$; that is, $\Delta_{01} a_{m n j k} \leq 0$ and $\Delta_{10} a_{m n j k} \leq 0$ for every $j, k=0,1, \ldots$. The nonincreasing case is defined analogously.
Recently, Nagy [13] did some research on the approximation by Nörlund means of double Walsh-Fourier series for Lipschitz functions and generalized Theorems A and B to the functions of two variables. We present one of the main results in [13] here.

Theorem C. Let $f \in \operatorname{Lip}(\alpha, p)$ for some $\alpha>0$ and $1 \leq p \leq$ $\infty$; let $\left\{q_{j k}\right\}$ be a double sequence of nonnegative numbers such that it is nondecreasing; $\Delta_{11} q_{j k}$ is of fixed sign and satisfies the regularity condition:

$$
\begin{gathered}
\frac{(m+1)(n+1) q_{m n}}{Q_{m n}}=\mathcal{O}(1) . \\
Q_{m n}:=\sum_{i=0}^{m} \sum_{j=0}^{m} q_{i j} \quad(m, n=0,1, \ldots),
\end{gathered}
$$

then,

$$
\begin{aligned}
& \left\|W_{m n}(f)-f\right\|_{L_{p}} \\
& \leq \begin{cases}\mathcal{O}\left(m^{-\alpha}+n^{-\alpha}\right), & 0<\alpha<1, \\
\mathcal{O}\left(m^{-1} \log m+n^{-1} \log n\right), & \alpha=1, \\
\mathcal{O}\left(m^{-1}+n^{-1}\right), & \alpha>1,\end{cases}
\end{aligned}
$$

where

$$
W_{m n}(f, x, y):=\frac{1}{Q_{m n}} \sum_{i=0}^{m} \sum_{j=0}^{m} q_{m n, m-i, n-j} S_{i j}(x, y),
$$

and $S_{i j}(x, y)$ is defined as in (16).

We know that in the theory of Fourier series it is of main interest how to approximate the function from the partial sums of its Fourier series. The purpose of the present paper is to get the rate of uniform approximation by $T$ transformation with doubly triangular. We give the outline of the paper. In Section 2, we state the main results. Some auxiliary lemmas are given in Section 3, and the proofs of the main theorems are presented in Section 4. Our new results can be applied to many classical summability methods such as Nörlund summability and Riesz summability. As an important application, we will apply them to the Nörlund summability and weighted means in Section 5. We will see that not only Theorems A, B, and C are corollaries of our results but also some other new types of estimates are presented in this paper.

\section{The Main Results}

For a fixed $n, \alpha_{n}:=\left\{a_{n k}\right\}$ of nonnegative numbers tending to zero is called rest bounded variation, or briefly $\alpha_{n} \in$ RBVS, if there is a constant $K\left(\alpha_{n}\right)$, only depending on $\alpha_{n}$, such that

$$
\sum_{k=m}^{\infty}\left|a_{n k}-a_{n, k+1}\right| \leq K\left(\alpha_{n}\right) a_{n m}
$$

holds for all natural numbers $m$. 
For a fixed $n, \alpha_{n}:=\left\{a_{n k}\right\}$ of nonnegative numbers tending to zero is called head bounded variation, or briefly $\alpha_{n} \in \mathrm{HBVS}$, if there is a constant $K\left(\alpha_{n}\right)$ only depending on $\alpha_{n}$ such that

$$
\sum_{k=0}^{m}\left|a_{n k}-a_{n, k+1}\right| \leq K\left(\alpha_{n}\right) a_{n m}
$$

for all natural numbers $m$, or only for all $m \leq N$ if the sequence $\alpha_{n}$ has only finite nonzero terms, and the last nonzero term is $a_{n N}$.

Remark A. The definitions of RBVS and HBVS are introduced by Leindler [14] to generalize the monotonicity conditions on sequences. In fact, RBVS and HBVS generalized monotone nonincreasing sequences and monotone nondecreasing sequences, respectively.

Remark $B$. Since it involves a sequence $K\left(\alpha_{n}\right)$, there should be a constant $K$ such that $0<K\left(\alpha_{n}\right) \leq K$.

Now, we extend the concepts of RBVS and HBVS to the double sequences as follows.

Definition 1. A double sequence $\alpha_{m n}=\left\{a_{m n j k}\right\}$ is called DRBVS, if there is a constant $K\left(\alpha_{m n}\right)$ such that for $j, k=$ $0,1,2, \ldots$,

$$
\left.\begin{array}{l}
\sum_{\mu=j}^{\infty}\left|\Delta_{10} a_{m n \mu k}\right| \\
\sum_{\nu=k}^{\infty}\left|\Delta_{01} a_{m n j \nu}\right| \\
\sum_{\mu=j}^{\infty} \sum_{\nu=k}^{\infty}\left|\Delta_{11} a_{m n j \nu}\right|
\end{array}\right\} \leq K\left(\alpha_{m n}\right) a_{m n j k} .
$$

Definition 2. A double sequence $\alpha_{m n}=\left\{a_{m n j k}\right\}$ is called DHBVS, if there is a constant $K\left(\alpha_{m n}\right)$ such that for $j, k=$ $0,1,2, \ldots$,

$$
\left.\begin{array}{c}
\sum_{\mu=0}^{j}\left|\Delta_{10} a_{m n \mu k}\right| \\
\sum_{\nu=0}^{k}\left|\Delta_{01} a_{m n j \nu}\right| \\
\sum_{\mu=0}^{j} \sum_{\nu=0}^{k}\left|\Delta_{11} a_{m n j \nu}\right|
\end{array}\right\} \leq K\left(\alpha_{m n}\right) a_{m n j k} .
$$

It is also required that the sequence $K\left(\alpha_{m n}\right)$ is bounded; that is, there is a positive constant $K$ such that $0<K\left(\alpha_{m n}\right) \leq$ $K$.

We state our main theorems as follows.
Theorem 3. Let $f \in L_{p}$, $\left\{t_{\text {mnuv }}\right\}$ be a a doubly normal triangular matrix of nonnegative numbers, $\left\{t_{\text {mпи }}\right\} \in D R B V S$, and let $E:=\lceil m\rceil, F:=\lceil n\rceil$. Then, one has

$$
\begin{aligned}
\| T_{m n} & -f \|_{p} \\
& \leq \mathscr{C} \sum_{k=0}^{E-1} \sum_{l=0}^{F-1} 2^{k+l} t_{m n 2^{k} 2^{l}}\left(\omega^{p}\left(f, 2^{-k}\right)+\omega^{p}\left(f, 2^{-l}\right)\right) .
\end{aligned}
$$

Also, if $f \in L_{p},\left\{t_{m n u v}\right\}$ is doubly normal triangular of nonnegative numbers; $\left\{t_{\text {mmuv }}\right\} \in D H B V S$; then one has the conclusion as follows:

$$
\begin{aligned}
\| T_{m n}- & f \|_{p} \\
\leq & \mathscr{C} \sum_{k=0}^{E-1} \sum_{l=0}^{F-1} 2^{k+l} t_{m n\left(2^{k+1}-1\right)\left(2^{l+1}-1\right)} \\
& \times\left(\omega^{p}\left(f, 2^{-k}\right)+\mathscr{C} \omega^{p}\left(f, 2^{-l}\right)\right) \\
+ & \mathscr{C} \sum_{k=0}^{E-1} 2^{k}\left(n-2^{F}\right) t_{m n\left(2^{k+1}-1\right) n} \\
& \times\left(\omega^{p}\left(f, 2^{-k}\right)+\omega^{p}\left(f, 2^{-F}\right)\right) \\
+ & \mathscr{C} \sum_{l=0}^{F-1} 2^{l}\left(m-2^{E}\right) t_{m n m\left(2^{2+1}-1\right)} \\
& \times\left(\omega^{p}\left(f, 2^{-E}\right)+\omega^{p}\left(f, 2^{-l}\right)\right) \\
+ & \mathscr{C}\left(m-2^{E}\right)\left(n-2^{F}\right) t_{m n m n} \\
& \times\left(\omega^{p}\left(f, 2^{-E}\right)+\omega^{p}\left(f, 2^{-F}\right)\right) .
\end{aligned}
$$

Theorem 4. Suppose that $f \in \operatorname{Lip}(p, \alpha)$ for $\alpha>0,1 \leq p \leq$ $+\infty$. Let $\left\{t_{m n u v}\right\}$ be nonnegative, doubly normal triangular, and $\left\{t_{m n u v}\right\} \in D H B V S$, satisfying $m n t_{m n m n}=\mathcal{O}(1)$. Then, one has

$$
\left\|T_{m n}-f\right\|_{p}= \begin{cases}\mathcal{O}\left(m^{-\alpha}+n^{-\alpha}\right), & 0<\alpha<1, \\ \mathcal{O}\left(m^{-1} \log m+n^{-1} \log n\right), & \alpha=1, \\ \mathcal{O}\left(m^{-1}+n^{-1}\right), & \alpha>1 .\end{cases}
$$

While for $f \in \operatorname{Lip}(p, \alpha)$ and for $\alpha>0,1 \leq p \leq$ $+\infty$, let $\left\{t_{m n u v}\right\}$ be nonnegative, normal doubly triangular, and $\left\{t_{\text {mnuv }}\right\} \in D R B V S$; one has

$$
\left\|T_{m n}-f\right\|_{p}=\mathcal{O}(1) \sum_{k=0}^{E-1} \sum_{l=0}^{F-1}\left(2^{k(1-\alpha)+l}+2^{k+l(1-\alpha)}\right) t_{m n 2^{k} 2^{l}} .
$$




\section{Lemmas and Proofs}

Lemma 1. Suppose that $\left\{t_{m n u v}\right\}$ is doubly normal triangular and $D_{u}$ is defined as (4); write $E:=\lceil m\rceil, F:=\lceil n\rceil$; then one has

$$
\begin{gathered}
A_{1}:=\sum_{u=0}^{2^{E}-1} \sum_{v=0}^{2^{F}-1} t_{m n u v} D_{u}(s) D_{v}(t) \\
=\sum_{k=0}^{E-1} \sum_{l=0}^{F-1} w_{2^{k+1}-1}(s) w_{2^{l+1}-1}(t) \\
\times\left(2^{k+l} t_{m n 2^{k} 2^{l} K_{2^{k}}(s) K_{2^{l}}(t)}\right. \\
+\sum_{i=1}^{2^{k}-1} i 2^{l} \Delta_{10} t_{m n\left(2^{k+1}-i\right) 2^{l}} K_{i}(s) K_{2^{l}}(t) \\
+\sum_{j=1}^{2^{l}-1} j 2^{k} \Delta_{01} t_{m n 2^{k}\left(2^{l+1}-j\right)} K_{j}(t) K_{2^{k}}(s) \\
+\sum_{i=1}^{2^{k}-1} \sum_{j=1}^{2^{l}-1} \Delta_{11} t_{m n\left(2^{k+1}-i\right)\left(2^{l+1}-j\right)} \\
\left.+i j K_{i}(s) K_{j}(t)\right)
\end{gathered}
$$$$
-\sum_{k=0}^{E-1} \sum_{l=0}^{F-1} w_{2^{k+1}-1}(s) D_{2^{l+1}}(t)
$$$$
\times\left(2^{k} K_{2^{k}}(s) \sum_{j=0}^{2^{l}-1} t_{m n 2^{k}\left(2^{l}+j\right)}\right.
$$$$
\left.+\sum_{i=1}^{2^{k}-1} \sum_{j=0}^{2^{l}-1} \Delta_{10} t_{m n\left(2^{k+1}-i\right)\left(2^{l}+j\right)} i K_{i}(s)\right)
$$$$
-\sum_{k=0}^{E-1} \sum_{l=0}^{F-1} D_{2^{k+1}}(s) w_{2^{l+1}-1}(t)
$$$$
\times\left(2^{l} K_{2^{l}}(t) \sum_{i=0}^{2^{k}-1} t_{m n\left(2^{k}+i\right) 2^{l}}\right.
$$$$
\left.+\sum_{i=0}^{2^{k}-1} \sum_{j=1}^{2^{l}-1} \Delta_{01} t_{m n\left(2^{k}+i\right)\left(2^{l+1}-j\right)} j K_{j}(s)\right)
$$$$
+\sum_{k=0}^{E-1} \sum_{l=0}^{F-1} \sum_{i=0}^{2^{k}-1} \sum_{j=0}^{2^{l}-1} t_{m n\left(2^{k}+i\right)\left(2^{l}+j\right)} D_{2^{k+1}}(s) D_{2^{l+1}}(t) .
$$

Proof. We can rewrite $A_{1}$ as

$$
A_{1}=\sum_{k=0}^{E-1} \sum_{l=0}^{F-1} \sum_{i=0}^{2^{k}-1} \sum_{j=0}^{2^{l}-1} t_{m n\left(2^{k}+i\right)\left(2^{l}+j\right)} D_{2^{k}+i}(s) D_{2^{l}+j}(t)
$$

and keep dividing the above into 4 parts:

$$
\begin{aligned}
A_{1}= & \sum_{k=0}^{E-1} \sum_{l=0}^{F-1} \sum_{i=0}^{2^{k}-1} \sum_{j=0}^{2^{l}-1} t_{m n\left(2^{k}+i\right)\left(2^{l}+j\right)}\left(D_{2^{k}+i}(s)-D_{2^{k+1}}(s)\right) \\
& \times\left(D_{2^{l}+j}(t)-D_{2^{l+1}}(t)\right) \\
& +\sum_{k=0}^{E-1} \sum_{l=0}^{F-1} \sum_{i=0}^{2^{k}-1} \sum_{j=0}^{2^{l}-1} t_{m n\left(2^{k}+i\right)\left(2^{l}+j\right)} \\
& +\sum_{k=0}^{E-1} \sum_{1=0}^{F-1} \sum_{i=0}^{2^{k}-1} \sum_{j=0}^{2^{l}-1} t_{m n\left(2^{k}+i\right)\left(2^{l}+j\right)} \\
& +\sum_{k=0}^{E-1} \sum_{l=0}^{F-1} \sum_{i=0}^{2^{k}-1} \sum_{j=0}^{2^{l}-1} t_{m n\left(2^{k}+i\right)\left(2^{l}+j\right)} D_{2^{k+1}}(s) D_{2^{l+1}}(t) \\
:= & \left.A_{11}+A_{12}+D_{2^{k+1}}(s)\right) D_{2^{l+1}}(t) \\
& +A_{14} .
\end{aligned}
$$

By using (8), we have

$$
\begin{gathered}
A_{11}=\sum_{k=0}^{E-1} \sum_{l=0}^{F-1} \sum_{i=0}^{2^{k}-1} \sum_{j=0}^{2^{l}-1} t_{m n\left(2^{k}+i\right)\left(2^{l}+j\right)} w_{2^{k+1}-1}(s) \\
\times D_{2^{k}-i}(s) w_{2^{l+1}-1}(t) D_{2^{l}-j}(t) \\
=\sum_{k=0}^{E-1} \sum_{l=0}^{F-1} \sum_{i=1}^{2^{k}} \sum_{j=1}^{2^{l}} t_{m n\left(2^{k+1}-i\right)\left(2^{l+1}-j\right)} w_{2^{k+1}-1}(s) \\
\times D_{i}(s) w_{2^{l+1}-1}(t) D_{j}(t) .
\end{gathered}
$$

Applying double Abel's transformation,

$$
\begin{aligned}
A_{11}=\sum_{k=0}^{E-1} \sum_{l=0}^{F-1} w_{2^{k+1}-1}(s) w_{2^{l+1}-1}(t) \\
\quad \times\left(\sum_{i_{1}=1}^{2^{k}} \sum_{j_{1}=1}^{2^{l}} D_{i_{1}}(s) D_{j_{1}}(t) t_{m n 2^{k} 2^{l}}\right. \\
+\sum_{j_{1}=1}^{2^{l}} D_{j_{1}}(t) \sum_{i=1}^{2^{k}-1} \Delta_{10} t_{m n\left(2^{k+1}-i\right) 2^{l}}
\end{aligned}
$$




$$
\begin{aligned}
& \times \sum_{i_{1}=1}^{i} D_{i_{1}}(s) \\
& +\sum_{i_{1}=1}^{2^{k}} D_{i_{1}}(s) \sum_{j=1}^{2^{l}-1} \Delta_{01} t_{m n 2^{k}\left(2^{l+1}-j\right)} \\
& \times \sum_{j_{1}=1}^{j} D_{j_{1}}(t) \\
& +\sum_{i=1}^{2^{k}-1} \sum_{j=1}^{2^{l}-1} \Delta_{11} t_{m n\left(2^{k+1}-i\right)\left(2^{l+1}-j\right)} \\
& \left.\times \sum_{i_{1}=1}^{i} D_{i_{1}}(s) \sum_{j_{1}=1}^{j} D_{j_{1}}(t)\right) .
\end{aligned}
$$

Next, (5) leads to

$$
\begin{aligned}
A_{11}=\sum_{k=0}^{E-1} \sum_{l=0}^{F-1} w_{2^{k+1}-1}(s) w_{2^{l+1}-1}(t) \\
\times\left(2^{k+l} t_{m n 2^{k} 2^{l}} K_{2^{k}}(s) K_{2^{l}}(t)\right. \\
+\sum_{i=1}^{2^{k}-1} i 2^{l} \Delta_{10} t_{m n\left(2^{k+1}-i\right) 2^{l}} K_{i}(s) K_{2^{l}}(t) \\
+\sum_{j=1}^{2^{l}-1} j 2^{k} \Delta_{01} t_{m n 2^{k}\left(2^{l+1}-j\right)} K_{j}(t) K_{2^{k}}(s) \\
+\sum_{i=1}^{2^{k}-1} \sum_{j=1}^{2^{l}-1} \Delta_{11} t_{m n\left(2^{k+1}-i\right)\left(2^{l+1}-j\right)} \\
\end{aligned}
$$

Using (8), (5), and Abel's transformation, similar to the estimate of $A_{11}$ (more easily actually),

$$
\begin{aligned}
A_{12}= & -\sum_{k=0}^{E-1} \sum_{l=0}^{F-1} w_{2^{k+1}-1}(s) D_{2^{l+1}}(t) 2^{k} K_{2^{k}}(s) \\
& \times \sum_{j=0}^{2^{l}-1} t_{m n 2^{k}\left(2^{l}+j\right)} \\
-\sum_{k=0}^{E-1} \sum_{l=0}^{F-1} w_{2^{k+1}-1}(s) D_{2^{l+1}} & \\
& \times \sum_{i=1}^{2^{k}-1} \sum_{j=0}^{2^{l}-1} \Delta_{10} t_{m n\left(2^{k+1}-i\right)\left(2^{l}+j\right)} i K_{i}(s) .
\end{aligned}
$$

Analogously,

$$
\begin{aligned}
A_{13}=-\sum_{k=0}^{E-1} \sum_{l=0}^{F-1} w_{2^{l+1}-1}(t) D_{2^{k+1}}(s) 2^{l} K_{2^{l}}(t) & \\
& \times \sum_{i=0}^{2^{k}-1} t_{m n\left(2^{k}+i\right) 2^{l}} \\
- & \sum_{k=0}^{E-1} \sum_{l=0}^{F-1} D_{2^{k+1}} w_{2^{k+1}-1}(s) \\
& \times \sum_{i=0}^{2^{k}-1} \sum_{j=1}^{2^{l}-1} \Delta_{10} t_{m n\left(2^{k}+i\right)\left(2^{l+1}-j\right)} j K_{j}(s) .
\end{aligned}
$$

Combining the above estimates of $A_{1 i}(i=1,2,3,4)$, we obtain the conclusion of Lemma 1.

Lemma 2. Let $\left\{t_{m n u v}\right\}$ be the same matrix as in Lemma 1; then one has

$$
\begin{aligned}
& A_{2}:=\sum_{u=0}^{2^{E}-1} \sum_{v=2^{F}}^{n} t_{m n u v} D_{u}(s) D_{v}(t) \\
& =-\sum_{k=0}^{E-1} \sum_{j=0}^{n-2^{F}} w_{2^{k+1}-1}(s) D_{2_{F}}(t) \\
& \times\left(2^{k} K_{2^{k}}(s) t_{m n 2^{k}\left(2^{F}+j\right)}\right. \\
& \left.+\sum_{i=1}^{2^{k}-1} \Delta_{10} t_{m n\left(2^{k+1}-i\right)\left(2^{F}+j\right)} i K_{i}(s)\right) \\
& -\sum_{k=0}^{E-1} w_{2^{k+1}-1} r_{F}(t) \\
& \times\left(t_{m n 2^{k} n^{2}} 2^{k}\left(n-2^{F}\right) K_{2^{k}}(s) K_{n-2^{F}}(t)\right. \\
& +\sum_{i=0}^{2^{k}-1} \Delta_{10} t_{m n\left(2^{k+1}-i\right) n} i\left(n-2^{F}\right) \\
& \times K_{i}(s) K_{n-2^{F}}(t) \\
& +\sum_{j=0}^{n-2^{F}-1} \Delta_{01} t_{m n 2^{k}\left(2^{F}+j\right)} 2^{k} j K_{2^{k}}(s) K_{j}(t) \\
& +\sum_{i=1}^{2^{k}-1} \sum_{j=0}^{n-2^{F}-1} \Delta_{11} t_{m n\left(2^{k+1}-i\right)\left(2^{F}+j\right)} \\
& \left.\times i j K_{i}(s) K_{j}(t)\right)
\end{aligned}
$$




$$
\begin{array}{cc}
+\sum_{k=0}^{E-1} \sum_{i=0}^{2^{k}-1} \sum_{j=0}^{n-2^{F}} t_{m n\left(2^{k}+i\right)\left(2^{F}+j\right)} D_{2^{k+1}}(s) D_{2^{F}}(t) & -\sum_{l=0}^{F-1} r_{E}(s) D_{2^{l+1}}(t) \\
-\sum_{k=0}^{E-1} D_{2^{k+1}}(s) r_{F}(t) & \times\left(\sum_{j=0}^{2^{l}-1} t_{m n m\left(2^{l}+j\right)}\left(m-2^{E}\right) K_{m-2^{E}}(s)\right. \\
\times\left(\sum_{i=0}^{2^{k}-1} t_{m n\left(2^{k}+i\right) n}\left(n-2^{F}\right) K_{n-2^{F}}(t)\right. & \left.+\sum_{j=0}^{2^{l}-1} \sum_{i=0}^{m-2^{A}-1} \Delta_{10} t_{m n\left(2^{E}+i\right)\left(2^{l}+j\right)} i K_{i}(s)\right) .
\end{array}
$$$$
\left.+\sum_{i=0}^{2^{k}-1} \sum_{j=0}^{n-2^{F}-1} \Delta_{01} t_{m n\left(2^{k}+i\right)\left(2^{F}+j\right)} j K_{j}(t)\right) .
$$

Proof. Rewrite $A_{2}$ by some different decomposition method; combining the decomposition we used in Lemma 1, it follows that

Meanwhile,

$$
\begin{aligned}
& A_{3}:=\sum_{u=2^{E}}^{m} \sum_{v=0}^{2^{F}-1} t_{m n u v} D_{u}(s) D_{v}(t) \\
& =-\sum_{l=0}^{F-1} \sum_{i=0}^{m-2^{E}} w_{2^{l+1}-1}(t) D_{2_{E}}(s) \\
& \times\left(2^{l} K_{2^{l}}(t) t_{m n\left(2^{E}+i\right) 2^{l}}\right. \\
& \left.+\sum_{j=1}^{2^{l}-1} \Delta_{01} t_{m n\left(2^{E}+i\right)\left(2^{l+1}-j\right)} j K_{j}(t)\right) \\
& -\sum_{l=0}^{F-1} w_{2^{l+1}-1}(t) r_{E}(s) \\
& \times\left(t_{m n m 2^{l}} 2^{l}\left(m-2^{E}\right) K_{2^{l}}(t) K_{m-2^{E}}(s)\right. \\
& +\sum_{i=0}^{m-2^{E}-1} \Delta_{10} t_{m n\left(2^{E}+i\right) 2^{2}} i 2^{l} K_{i}(s) K_{2^{l}}(t) \\
& +\sum_{j=1}^{2^{l}-1} \Delta_{01} t_{m n m\left(2^{l+1}-j\right)}\left(m-2^{E}\right) \\
& \times j K_{m-2^{E}}(s) K_{j}(t) \\
& +\sum_{i=0}^{m-2^{E}-1} \sum_{j=1}^{2^{l}-1} \Delta_{11} t_{m n\left(2^{E}+i\right)\left(2^{l+1}-j\right)} \\
& \left.\times i j K_{i}(s) K_{j}(t)\right) \\
& -\sum_{l=0}^{F-1} \sum_{j=0}^{2^{l}-1} \sum_{i=0}^{m-2^{E}} t_{m n\left(2^{E}+i\right)\left(2^{l}+j\right)} D_{2^{E}}(s) D_{2^{l+1}}(t)
\end{aligned}
$$

$$
\begin{aligned}
A_{2}= & \sum_{k=0}^{E-1} \sum_{i=0}^{2^{k}-1} \sum_{v=2^{F}}^{n} t_{m n\left(2^{k}+i\right) v} D_{2^{k}+i}(s) D_{v}(t) \\
= & \sum_{k=0}^{E-1} \sum_{i=0}^{2^{k}-1} \sum_{j=0}^{n-2^{F}} t_{m n\left(2^{k}+i\right)\left(2^{F}+j\right)} D_{2^{k}+i}(s) D_{2^{F}+j}(t) \\
= & \sum_{k=0}^{E-1} \sum_{i=0}^{2^{k}-1} \sum_{j=0}^{n-2^{F}} t_{m n\left(2^{k}+i\right)\left(2^{F}+j\right)} \times\left(D_{2^{k}+i}(s)-D_{2^{k+1}}(s)\right) D_{2^{F}+j}(t) \\
& +\sum_{k=0}^{E-1} \sum_{i=0}^{2^{k}-1} \sum_{j=0}^{n-2^{F}} t_{m n\left(2^{k}+i\right)\left(2^{F}+j\right)} D_{2^{k+1}}(s) D_{2^{F}+j}(t) .
\end{aligned}
$$

Furthermore, by properties (7) and (8) of $D_{n}$, we have

$$
\begin{gathered}
A_{2}=\sum_{k=0}^{E-1} \sum_{i=0}^{2^{k}-1} \sum_{j=0}^{n-2^{F}} t_{m n\left(2^{k}+i\right)\left(2^{F}+j\right)}\left(-w_{2^{k+1}-1}(s) D_{2^{k}-i}(s)\right) \\
\quad \times\left(D_{2^{F}}(t)+r_{F}(t) D_{j}(t)\right) \\
+\sum_{k=0}^{E-1} \sum_{i=0}^{2^{k}-1} \sum_{j=0}^{n-2^{F}} t_{m n\left(2^{k}+i\right)\left(2^{F}+j\right)} D_{2^{k+1}}(s) \\
\quad \times\left(D_{2^{F}}(t)+r_{F}(t) D_{j}(t)\right) \\
\sum_{k=0}^{E-1} \sum_{i=0}^{2^{k}-1} \sum_{j=0}^{n-2^{F}} t_{m n\left(2^{k}+i\right)\left(2^{F}+j\right)} \\
\times\left(-D_{2^{F}}(t) w_{2^{k+1}-1}(s) D_{2^{k}-i}(s)\right. \\
-w_{2^{k+1}-1}(s) D_{2^{k}-i}(s) r_{F}(t) D_{j}(t) \\
+D_{2^{k+1}}(s) D_{2^{F}}(t) \\
\left.+D_{2^{k+1}}(s) r_{F}(t) D_{j}(t)\right) \\
A_{21}+A_{24} \cdot
\end{gathered}
$$


By changing $D_{2^{f}-i}$ to $D_{i}$ and applying Abel's transformation and (5),

$$
\begin{array}{r}
A_{21}=-\sum_{k=0}^{E-1} \sum_{i=1}^{2^{k}} \sum_{j=0}^{n-2^{F}} t_{m n\left(2^{k+1}-i\right)\left(2^{F}+j\right)} D_{2^{F}}(t) w_{2^{k+1}-1}(s) D_{i}(s) \\
=-\sum_{k=0}^{E-1} \sum_{j=0}^{n-2^{F}} w_{2^{k+1}-1}(s) D_{2^{F}}(t) \\
\times\left(2^{k} K_{2^{k}}(s) t_{m n 2^{k}\left(2^{F}+j\right)}\right. \\
\left.+\sum_{i=1}^{2^{k}-1} \Delta_{10} t_{m n\left(2^{k+1}-i\right)\left(2^{F}+j\right)} i K_{i}(s)\right) .
\end{array}
$$

Similarly,

$$
\begin{aligned}
A_{22}=-\sum_{k=0}^{E-1} w_{2^{k+1}-1} r_{F}(t) & \\
& \times\left(t_{m n 2^{k} n} 2^{k}\left(n-2^{F}\right) K_{2^{k}}(s) K_{n-2^{F}}(t)\right. \\
& +\sum_{i=0}^{2^{k}-1} \Delta_{10} t_{m n\left(2^{k+1}-i\right) n} i\left(n-2^{F}\right) \\
& +\sum_{j=0}^{n-2^{F}-1} \Delta_{01} t_{m n 2^{k}\left(2^{F}+j\right)} 2^{k} j K_{2^{k}}(s) K_{j}(t) \\
& +\sum_{i=1}^{2^{k}-1} \sum_{j=0}^{n-2^{F}-1} \Delta_{11} t_{m n\left(2^{k+1}-i\right)\left(2^{F}+j\right)} \\
& \left.\times i j K_{i}(s) K_{j}(t)\right) .
\end{aligned}
$$

We also obtain the estimate for

$$
\begin{aligned}
A_{24}=-\sum_{k=0}^{E-1} D_{2^{k+1}}(s) r_{F}(t) \\
\quad \times\left(\sum_{i=0}^{2^{k}-1} t_{m n\left(2^{k}+i\right) n}\left(n-2^{F}\right) K_{n-2^{F}}(t)\right. \\
\\
\left.\quad+\sum_{i=0}^{2^{k}-1} \sum_{j=0}^{n-2^{F}-1} \Delta_{01} t_{m n\left(2^{k}+i\right)\left(2^{F}+j\right)} j K_{j}(t)\right) .
\end{aligned}
$$

Combining the estimates of $A_{2 i}(i=1,2,3,4)$, we have the conclusion for $A_{2}$ in Lemma 2 , and the discussion for $A_{3}$ is similar (as for the decomposition of $A_{2}$, we denote $A_{3}=$ $\sum_{i=1}^{4} A_{3 i}$ ). This completes the proof of Lemma 2.

Lemma 3. Let $\left\{t_{m n u v}\right\}$ be the same matrix as in Lemma 1. Then, one has

$$
\begin{aligned}
A_{4}:= & \sum_{u=2^{E}}^{m} \sum_{v=2^{F}}^{n} t_{m n u v} D_{u}(s) D_{v}(t) \\
= & \sum_{i=0}^{m-2^{E}} \sum_{j=0}^{n-2^{F}} t_{m n\left(2^{E}+i\right)\left(2^{F}+j\right)} D_{2^{E}}(s) D_{2^{F}}(t) \\
& +\sum_{i=0}^{m-2^{E}} D_{2^{E}}(s) r_{F}(t)
\end{aligned}
$$

$$
\begin{aligned}
& \times\left(t_{m n\left(2^{E}+i\right) n}\left(n-2^{F}\right) K_{n-2^{F}}(t)\right. \\
& \left.\quad+\sum_{j=0}^{n-2^{F}-1} \Delta_{01} t_{m n\left(2^{E}+i\right)\left(2^{F}+j\right)} j K_{j}(t)\right)
\end{aligned}
$$$$
+\sum_{j=0}^{n-2^{F}} r_{E}(s) D_{2^{F}}(t)
$$$$
\times\left(t_{m n m\left(2^{F}+j\right)}\left(m-2^{E}\right) K_{m-2^{E}}(s)\right.
$$$$
\left.+\sum_{i=0}^{m-2^{E}-1} \Delta_{10} t_{m n\left(2^{E}+i\right)\left(2^{F}+j\right)} i K_{i}(s)\right)
$$$$
+r_{E}(s) r_{F}(t)
$$$$
\times\left(t_{m n m n}\left(m-2^{E}\right)\left(n-2^{F}\right) K_{m-2^{E}}(s) K_{n-2^{F}}(t)\right.
$$$$
+\sum_{i=0}^{m-2^{E}-1} \Delta_{10} t_{m n\left(2^{E}+i\right) n} i\left(n-2^{F}\right) K_{i}(s) K_{n-2^{F}}(t)
$$$$
+\sum_{j=0}^{n-2^{F}-1} \Delta_{01} t_{m n m\left(2^{F}+j\right)} j\left(m-2^{E}\right) K_{m-2^{E}}(s) K_{j}(t)
$$$$
\left.+\sum_{i=0}^{m-2^{E}-1} \Delta_{11} t_{m n\left(2^{E}+i\right)\left(2^{F}+j\right)} i j K_{i}(s) K_{j}(t)\right) .
$$

Proof. Decompose $A_{4}$ into 4 parts:

$$
A_{4}=\sum_{i=0}^{m-2^{E}} \sum_{j=0}^{n-2^{F}} t_{m n\left(2^{E}+i\right)\left(2^{F}+j\right)} D_{2^{E}+i}(s) D_{2^{F}+j}(t)
$$




$$
\begin{aligned}
= & \sum_{i=0}^{m-2^{E}} \sum_{j=0}^{n-2^{F}} t_{m n\left(2^{E}+i\right)\left(2^{F}+j\right)}\left(D_{2^{E}}(s)+r_{E}(s) D_{i}(s)\right) \\
& \times\left(D_{2^{F}}(t)+r_{F}(t) D_{j}(t)\right) \\
= & \sum_{i=0}^{m-2^{E}} \sum_{j=0}^{n-2^{F}} t_{m n\left(2^{E}+i\right)\left(2^{F}+j\right)} D_{2^{E}}(s) D_{2^{F}}(t) \\
& +\sum_{i=0}^{m-2^{E}} \sum_{j=0}^{n-2^{F}} t_{m n\left(2^{E}+i\right)\left(2^{F}+j\right)} D_{2^{E}}(s) r_{F}(t) D_{j}(t) \\
& +\sum_{i=0}^{m-2^{E}} \sum_{j=0}^{n-2^{F}} t_{m n\left(2^{E}+i\right)\left(2^{F}+j\right)} r_{E}(s) D_{i}(s) D_{2^{F}}(t) \\
& +\sum_{i=0}^{m-2^{E}} \sum_{j=0}^{n-2^{F}} t_{m n\left(2^{E}+i\right)\left(2^{F}+j\right)} r_{E}(s) r_{F}(t) D_{i}(s) D_{j}(t) \\
= & A_{41}+A_{42}+A_{43}+A_{44} \cdot
\end{aligned}
$$

Using the techniques as in Lemmas 1 and 2, we can easily get the result of Lemma 3 .

By Lemmas 1, 2, and 3 and (20), $K_{m n}(s, t)$ can be written as

$$
\begin{aligned}
K_{m n}(s, t) & =\sum_{u=0}^{m} \sum_{v=0}^{n} t_{m n u v} D_{u}(s) D_{v}(t) \\
& =\sum_{i=1}^{4} A_{i}=\sum_{i=1}^{4} \sum_{j=1}^{4} A_{i j} .
\end{aligned}
$$

Therefore, by (21), we have

$$
\begin{aligned}
& T_{m n}(x, y)-f(x, y) \\
& \quad=\iint_{0}^{1} \sum_{i=1}^{4} \sum_{j=1}^{4} A_{i j}(f(x+s, y+t)-f(x, y)) d s d t .
\end{aligned}
$$

We denote by $\mathscr{P}_{n}$ the set of Walsh polynomials of order less than $n$; that is,

$$
\mathscr{P}_{n}:=\left\{P(x), P(x):=\sum_{i=0}^{n-1} c_{i} w_{i}(x)\right\},
$$

where $n \geq 1$ and $c_{i}$ denotes the real or complex numbers. On the torus $I^{2}$, we define the two-dimensional Walsh polynomials of order less than $(m, n)$ as

$$
\mathscr{P}_{m, n}:=\left\{P_{1}(s) \times P_{2}(t), P_{1} \in \mathscr{P}_{m}, P_{2} \in \mathscr{P}_{n}, s, t \in I\right\} .
$$

\section{Lemma 4. Consider}

$$
\begin{aligned}
\Theta & :=\left\|\int_{I^{2}} D_{2^{k}}(s) D_{2^{l}}(t)(f(\cdot+s, \cdot+t)-f(\cdot, \cdot)) d s d t\right\|_{p} \\
& \leq \mathscr{C}\left(\omega^{p}\left(f, 2^{-k}\right)+\omega^{p}\left(f, 2^{-l}\right)\right) .
\end{aligned}
$$

Proof. By (6) and Hölder inequality, we have

$$
\begin{array}{r}
\Theta \leq\left\|\int_{I_{k} \times I_{l}} D_{2^{k}}(s) D_{2^{l}}(t)(f(\cdot+s, \cdot+t)-f(\cdot, \cdot)) d s d t\right\|_{p} \\
\leq\left[\int _ { I ^ { 2 } } \left(\int_{I_{k} \times I_{l}} D_{2^{k}}(s) D_{2^{l}}(t)\right.\right. \\
\left.\left.\quad \times(f(\cdot+s, \cdot+t)-f(\cdot, \cdot))^{p} d s d t\right) d x d y\right]^{1 / p} .
\end{array}
$$

Furthermore, using the generalized Minkowski inequality, we have

$$
\begin{aligned}
\Theta \leq & \int_{I_{k} \times I_{l}} D_{2^{k}}(s) D_{2^{l}}(t) \\
& \times\left(\int_{I^{2}}(f(\cdot+s, \cdot+t)-f(\cdot, \cdot))^{p} d x d y\right)^{1 / p} d s d t \\
\leq & \omega^{p}\left(f, 2^{-k}, 2^{-l}\right) \leq \mathscr{C}\left(\omega^{p}\left(f, 2^{-k}\right)+\omega^{p}\left(f, 2^{-l}\right)\right) .
\end{aligned}
$$

This completes the proof of Lemma 4.

Lemma 5. Let $P \in \mathscr{P}_{2^{k}, 2^{l}}, Q \in \mathscr{P}_{2^{k}}, 1 \leq p \leq \infty, k, l \in \mathbb{N}$; then one has

(i)

$$
\begin{aligned}
& \left\|\iint_{0}^{1}(f(\cdot+s, \cdot+t)-f(\cdot, \cdot)) r_{k}(s) r_{l}(t) P(s, t) d s d t\right\|_{P} \\
& \quad \leq C\|P\|_{1} \omega_{1,2}^{p}\left(f, 2^{-k}, 2^{-l}\right),
\end{aligned}
$$

(ii)

$$
\begin{aligned}
& \left\|\iint_{0}^{1}(f(\cdot+s, \cdot+t)-f(\cdot, \cdot)) r_{k}(s) D_{2^{l}}(t) Q(s) d s d t\right\|_{p} \\
& \quad \leq C\|Q\|_{1} \omega_{1,2}^{p}\left(f, 2^{-k}, 2^{-l}\right) .
\end{aligned}
$$

Proof. Note that $P \in \mathscr{P}_{2^{k}, 2^{l}}$ and $Q \in \mathscr{P}_{2^{k}}$ are constants on the sets $I_{k} \times I_{l}$ and $I_{k}$, respectively. By Lemma 4, it is not difficult to prove Lemma 5. We can also find the conclusions in [13].

\section{Lemma 6. Suppose that}

(i) $\left\{t_{m n u v}\right\} \in D R B V S$, and it is doubly triangular; then for any $i_{1} \geq i_{2}$ or $j_{1} \geq j_{2}$, one has $t_{m n i_{1} j}=\mathcal{O}\left(t_{m n i_{2} j}\right)$ or $t_{m n i j_{1}}=\mathcal{O}\left(t_{m n i j_{2}}\right)$.

(ii) $\left\{t_{m n u v}\right\} \in D H B V S$, and it is doubly triangular; then for any $i_{1} \leq i_{2}$ or $j_{1} \leq j_{2}$, one has $t_{m n i_{1} j}=\mathcal{O}\left(t_{m n i_{2} j}\right)$ or $t_{m n i j_{1}}=\mathcal{O}\left(t_{m n i j_{2}}\right)$.

Proof. (i) Since $\left\{t_{\text {mnuv }}\right\}$ is triangular,

$$
t_{m n i_{1} j} \leq \sum_{u=i_{1}}^{m}\left|\Delta_{10} t_{m n u j}\right| \leq \sum_{u=i_{2}}^{m}\left|\Delta_{10} t_{m m u j}\right|=\mathcal{O}\left(t_{m n i_{2} j}\right) .
$$


Similarly, we have

$$
t_{m n i j_{1}} \leq \sum_{v=j_{1}}^{m}\left|\Delta_{10} t_{m n i v}\right| \leq \sum_{v=j_{2}}^{m}\left|\Delta_{10} t_{m n i v}\right|=\mathcal{O}\left(t_{m n i j_{2}}\right) .
$$

(ii) Since

$$
\left|t_{m n i_{1} j}-t_{m n i_{2} j}\right| \leq \sum_{i=i_{1}}^{i_{2}}\left|\Delta_{10} t_{m n i j}\right|=\mathcal{O}\left(t_{m n i_{2} j}\right),
$$

it implies that

$$
t_{m n i_{1} j}=\mathcal{O}\left(t_{m n i_{2} j}\right)
$$

Similarly, we have

$$
t_{m n i j_{1}}=\mathcal{O}\left(t_{m n i j_{2}}\right)
$$

This completes the proof of Lemma 6.

\section{Proofs of Theorems 3 and 4}

We denote the norm of $\iint_{0}^{1} A_{i j}(f(x+s, y+t)-f(x, y)) d s d t$ by

$$
\Phi_{i j}:=\left\|\iint_{0}^{1} A_{i j}(f(\cdot+s, \cdot+t)-f(\cdot, \cdot)) d s d t\right\|_{p} .
$$

Then by (60) and Minkowski inequality, we have

$$
\left\|T_{m n}-f\right\|_{p} \leq \sum_{i=1}^{4} \sum_{j=1}^{4} \Phi_{i j}
$$

Proof of Theorem 3. (I) When $\left\{t_{m n i j}\right\} \in$ DRBVS, we deduce $\Phi_{i j}$ separately, by (9) and Lemma 5(i),

$$
\begin{aligned}
& \Phi_{11} \leq \mathscr{C} \sum_{k=0}^{E-1} \sum_{l=0}^{F-1} 2^{k+l} t_{m n 2^{k} 2^{l}} \omega_{1,2}^{p}\left(f, 2^{-k}, 2^{-l}\right) \\
&+\mathscr{C} \sum_{k=0}^{E-1} \sum_{l=0}^{F-1} \sum_{i=1}^{2^{k}-1} i 2^{l}\left|\Delta_{10} t_{m n\left(2^{k+1}-i\right) 2^{l}}\right| \omega_{1,2}^{p}\left(f, 2^{-k}, 2^{-l}\right) \\
&+\mathscr{C} \sum_{k=0}^{E-1} \sum_{l=0}^{F-1} \sum_{j=1}^{2^{l}-1} j 2^{k}\left|\Delta_{01} t_{m n 2^{k}\left(2^{l+1}-j\right)}\right| \omega_{1,2}^{p}\left(f, 2^{-k}, 2^{-l}\right) \\
&+\mathscr{C} \sum_{k=0}^{E-1} \sum_{l=0}^{F-1} \sum_{i=1}^{2^{k}-1} \sum_{j=1}^{2^{l}-1}\left|\Delta_{11} t_{m n\left(2^{k+1}-i\right)\left(2^{l+1}-j\right)}\right| \\
& \times i j \omega_{1,2}^{p}\left(f, 2^{-k}, 2^{-l}\right) .
\end{aligned}
$$

Since $\left\{t_{m n i j}\right\} \in$ DRBVS, by the definition of DRBVS, we have

$$
\begin{aligned}
& \sum_{i=1}^{2^{k}-1} i 2^{l}\left|\Delta_{10} t_{m n\left(2^{k+1}-i\right) 2^{l}}\right| \leq 2^{k+l} t_{m n 2^{k} 2^{l}}, \\
& \sum_{j=1}^{2^{l}-1} j 2^{k}\left|\Delta_{01} t_{m n 2^{k}\left(2^{l+1}-j\right)}\right| \leq 2^{k+l} t_{m n 2^{k} 2^{l}}, \\
& \sum_{i=1}^{2^{k}-1} \sum_{j=1}^{2^{l}-1}\left|\Delta_{11} t_{m n\left(2^{k+1}-i\right)\left(2^{l+1}-j\right)}\right| i j \leq 2^{k+l} t_{m n 2^{k} 2^{l}} .
\end{aligned}
$$

Substituting the above into $\Phi_{11}$ yields

$$
\Phi_{11} \leq \mathscr{C} \sum_{k=0}^{E-1} \sum_{l=0}^{F-1} 2^{k+l} t_{m n 2^{k} 2^{2}} \omega_{1,2}^{p}\left(f, 2^{-k}, 2^{-l}\right) .
$$

Now by (9) and Lemma 5(ii),

$$
\begin{aligned}
\Phi_{12} \leq & \mathscr{C} \sum_{k=0}^{E-1}\left(\sum_{l=0}^{F-1} \sum_{j=1}^{2^{l}-1} 2^{k} t_{m n 2^{k}\left(2^{l}+j\right)}\right. \\
& \left.+\sum_{l=0}^{F-1} \sum_{i=1}^{2^{k}-1} \sum_{j=1}^{2^{l}-1} i\left|\Delta_{10} t_{m n\left(2^{k+1}-i\right)\left(2^{l}+j\right)}\right|\right) \\
& \times \omega^{p}\left(f, 2^{-k}\right) .
\end{aligned}
$$

Applying Lemma 6(i) and still the definition of DRBVS, we have

$$
\begin{aligned}
\Phi_{12} \leq & \mathscr{C} \sum_{k=0}^{E-1} \sum_{l=0}^{F-1} 2^{k} \omega^{p}\left(f, 2^{-k}\right) \\
& \times\left(\sum_{j=1}^{2^{l}-1} t_{m n 2^{k}\left(2^{l}+j\right)}+t_{m n 2^{k} 2^{l}}\right) \\
& \leq \mathscr{C} \sum_{k=0}^{E-1} \sum_{l=0}^{F-1} 2^{k+l} \omega^{p}\left(f, 2^{-k}\right) t_{m n 2^{k} 2^{l}} .
\end{aligned}
$$

Analogously,

$$
\Phi_{13} \leq \mathscr{C} \sum_{k=0}^{E-1} \sum_{l=0}^{F-1} 2^{k+l} \omega^{p}\left(f, 2^{-l}\right) t_{m n 2^{k} 2^{l}}
$$

By Lemmas 4 and 6(i), it is easy to verify that

$$
\Phi_{14} \leq \mathscr{C} \sum_{k=0}^{E-1} \sum_{l=0}^{F-1} 2^{k+l} t_{m n 2^{k} 2^{l}}\left(\omega^{p}\left(f, 2^{-k}\right)+\omega^{p}\left(f, 2^{-l}\right)\right) .
$$


Similar discussion deduces that (by using Lemmas 4 and 5 (i) and (ii) separately)

$$
\begin{gathered}
\Phi_{21} \leq \mathscr{C} \sum_{k=0}^{E-1} 2^{k}\left(n-2^{F}\right) t_{m n 2^{k} 2^{F}} \omega^{p}\left(f, 2^{-k}\right), \\
\Phi_{22} \leq \mathscr{C}\left(\sum_{k=0}^{E-1} 2^{k}\left(n-2^{F}\right) t_{m n 2^{k} n}\right. \\
\left.+\sum_{k=0}^{E-1} 2^{k}\left(n-2^{F}\right) t_{m n 2^{k} 2^{F}}\right) \\
\times \omega_{23} \leq \omega^{p}\left(f, 2^{-k}, 2^{-F}\right), \\
\sum_{k=0}^{E-1} 2^{k}\left(n-2^{F}\right) t_{m n 2^{k} 2^{F}}\left(\omega^{p}\left(f, 2^{-k}\right)+\omega^{p}\left(f, 2^{-F}\right)\right), \\
\Phi_{24}^{E-1} \sum_{k=0}^{k}\left(n-2^{F}\right) t_{m n 2^{k} n} \omega^{p}\left(f, 2^{-F}\right) \\
+\mathscr{C} \sum_{k=0}^{E-1}\left(n-2^{F}\right) t_{m n 2^{k} 2^{F}} \omega^{p}\left(f, 2^{-F}\right) .
\end{gathered}
$$

Note the definition of $E$ and $F$; clearly, $m+1 \leq 2^{E+1}$ and $n+1 \leq 2^{F+1}$; thus we have

$$
\begin{aligned}
& m-2^{E} \leq 2^{E}-1=\sum_{k=0}^{E-1} 2^{k} \\
& n-2^{F} \leq 2^{F}-1=\sum_{l=0}^{F-1} 2^{l}
\end{aligned}
$$

Applying inequality (83) and Lemma 6 to (82), also using the monotonicity of continuous modulus, it is not difficult to deduce that

$$
\sum_{i=1}^{4} \Phi_{2 i} \leq \mathscr{C} \sum_{k=0}^{E-1} \sum_{l=0}^{F-1} 2^{k+l} t_{m n 2^{k} 2^{l}}\left(\omega^{p}\left(f, 2^{-k}\right)+\omega^{p}\left(f, 2^{-l}\right)\right)
$$

$\Phi_{3 i}(i=1,2,3,4)$ goes analogously as $\Phi_{2 i}(i=1,2,3,4) ;$ thus we have

$$
\sum_{i=1}^{4} \Phi_{3 i} \leq \mathscr{C} \sum_{k=0}^{E-1} \sum_{l=0}^{F-1} 2^{k+l} t_{m n 2^{k} 2^{l}}\left(\omega^{p}\left(f, 2^{-k}\right)+\omega^{p}\left(f, 2^{-l}\right)\right)
$$

Next, we estimate $\Phi_{4 i}, \quad(i=1,2,3,4)$. Applying Lemmas 4 and 6, we easily get

$$
\begin{aligned}
\Phi_{41} \leq & \mathscr{C}\left(m-2^{E}\right)\left(n-2^{F}\right) t_{m n 2^{E} 2^{F}} \\
& \times\left(\omega^{p}\left(f, 2^{-E}\right)+\omega^{p}\left(f, 2^{-F}\right)\right), \\
\Phi_{42} \leq & \mathscr{C} \omega^{p}\left(f, 2^{-F}\right) \\
& \times\left(\left(m-2^{E}\right)\left(n-2^{F}\right) t_{m n 2^{E} n}+\left(n-2^{F}\right) t_{m n 2^{E} 2^{F}}\right), \\
\Phi_{43} \leq & \mathscr{C} \omega^{p}\left(f, 2^{-E}\right) \\
& \times\left(\left(m-2^{E}\right)\left(n-2^{F}\right) t_{m n m 2^{F}}+\left(m-2^{E}\right) t_{m n 2^{E} 2^{F}}\right),
\end{aligned}
$$

and the last term

$$
\begin{aligned}
\Phi_{44} \leq & \mathscr{C} \omega^{p}\left(f, 2^{-E}, 2^{-F}\right)\left(m-2^{E}\right)\left(n-2^{F}\right) \\
& \times\left(t_{m n m n}+t_{m n 2^{E} n}+t_{m n m 2^{F}}+t_{m n 2^{E} 2^{F}}\right) .
\end{aligned}
$$

Applying Lemma 6(i) and (83) to (86) and (87), we have

$$
\sum_{i=1}^{4} \Phi_{4 i} \leq \mathscr{C} \sum_{k=0}^{E-1} \sum_{l=0}^{F-1} 2^{k+l} t_{m n 2^{k} 2^{l}}\left(\omega^{p}\left(f, 2^{-k}\right)+\omega^{p}\left(f, 2^{-l}\right)\right) .
$$

Combining all the estimates, (77)-(81), (84), (85), and (88) of $\Phi_{i j}$, yields that

$$
\begin{aligned}
\| T_{m n} & -f \|_{L_{p}} \\
& \leq \sum_{i=1}^{4} \sum_{j=1}^{4} \Phi_{i j} \\
& \leq \mathscr{C} \sum_{k=0}^{E-1} \sum_{l=0}^{F-1} 2^{k+l} t_{m n 2^{k} 2^{l}}\left(\omega^{p}\left(f, 2^{-k}\right)+\omega^{p}\left(f, 2^{-l}\right)\right) .
\end{aligned}
$$

(II) When $\left\{t_{m n i j}\right\} \in$ DHBVS, the proof goes similarly. We mainly point out the differences in this case and prove some terms of $\Phi_{i j}$ as examples.

Since $\left\{t_{m n i j}\right\} \in$ DHBVS, by the definition of DHBVS, we have

$$
\begin{aligned}
& \sum_{i=1}^{2^{k}-1} i 2^{l}\left|\Delta_{10} t_{m n\left(2^{k+1}-i\right) 2^{l}}\right| \leq 2^{k+l} t_{m n\left(2^{k+1}-1\right) 2^{l}}, \\
& \sum_{j=1}^{2^{l}-1} j 2^{k}\left|\Delta_{01} t_{m n 2^{k}\left(2^{l+1}-j\right)}\right| \leq 2^{k+l} t_{m n 2^{k}\left(2^{l+1}-1\right)}
\end{aligned}
$$

$\sum_{i=1}^{2^{k}-1} \sum_{j=1}^{2^{l}-1}\left|\Delta_{11} t_{m n\left(2^{k+1}-i\right)\left(2^{l+1}-j\right)}\right| i j \leq 2^{k+l} t_{m n\left(2^{k+1}-1\right)\left(2^{l+1}-1\right)}$.

Thus by Lemma 5(i), (9), (71), and (72), we have

$$
\Phi_{11} \leq \mathscr{C} \sum_{k=0}^{E-1} \sum_{l=0}^{F-1} 2^{k+l} t_{m n\left(2^{k+1}-1\right)\left(2^{l+1}-1\right)} \omega_{1,2}^{p}\left(f, 2^{-k}, 2^{-l}\right) .
$$


Lemma 5(ii), (9), (71), (72), and (83) yield that

$$
\Phi_{1 i} \leq \mathscr{C} \sum_{k=0}^{E-1} \sum_{l=0}^{F-1} 2^{k+l} t_{m n\left(2^{k+1}-1\right)\left(2^{l+1}-1\right)} \omega_{1,2}^{p}\left(f, 2^{-k}, 2^{-l}\right)
$$

Now the definition of DHBVS, Lemmas 5 and 6, and (9) deduce that

$$
\begin{aligned}
\Phi_{21} & \leq \mathscr{C} \sum_{k=0}^{E-1} 2^{k}\left(\left(n-2^{F}\right) t_{m n 2^{k} n}+t_{m n\left(2^{k+1}-1\right) n}\right) \omega^{p}\left(f, 2^{-k}\right) \\
& \leq \mathscr{C} \sum_{k=0}^{E-1} 2^{k}\left(n-2^{F}\right) t_{m n\left(2^{k+1}-1\right) n} \omega^{p}\left(f, 2^{-k}\right), \\
\Phi_{22} \leq & \mathscr{C} \sum_{k=0}^{E-1} 2^{k}\left(n-2^{F}\right) t_{m n\left(2^{k+1}-1\right) n} \omega^{p}\left(f, 2^{-k}, 2^{-F}\right), \\
\Phi_{23} \leq & \mathscr{C} \sum_{k=0}^{E-1} 2^{k}\left(n-2^{F}\right) t_{m n\left(2^{k+1}-1\right) n} \\
\Phi_{24} \leq & \mathscr{C} \sum_{k=0}^{E-1} 2^{k}\left(n-2^{F}\right) t_{m n\left(2^{k+1}-1\right) n} \omega^{p}\left(f, 2^{-F}\right) .
\end{aligned}
$$
have

While in this case we keep $t_{m n\left(2^{k+1}-1\right) n}$ in the sum, so we

$$
\begin{aligned}
\sum_{i=1}^{4} \Phi_{2 i} \leq \mathscr{C} \sum_{k=0}^{E-1} & 2^{k}\left(n-2^{F}\right) t_{m n\left(2^{k+1}-1\right) n} \\
& \times\left(\omega^{p}\left(f, 2^{-k}\right)+\omega^{p}\left(f, 2^{-F}\right)\right) .
\end{aligned}
$$

Analogously,

$$
\begin{aligned}
\sum_{i=1}^{4} \Phi_{3 i} \leq & \mathscr{C} \sum_{l=0}^{F-1}\left(m-2^{E}\right) 2^{l} t_{m n m\left(2^{l+1}-1\right)} \\
& \times\left(\omega^{p}\left(f, 2^{-E}\right)+\omega^{p}\left(f, 2^{-l}\right)\right), \\
\sum_{i=1}^{4} \Phi_{4 i} \leq & \mathscr{C}\left(m-2^{E}\right)\left(n-2^{F}\right) t_{m n m n} \\
& \times\left(\omega^{p}\left(f, 2^{-E}\right)+\omega^{p}\left(f, 2^{-F}\right)\right) .
\end{aligned}
$$

Combining all the estimates of (92)-(96), we obtain the conclusion of the case of $\left\{t_{m n i j}\right\} \in$ DHBVS in Theorem 3 .
Remark C. Actually, in the case of $\left\{t_{m n i j}\right\} \in$ DHBVS, (96) can be represented in a more complicated form if we calculate the term with the same manner. For instance,

$$
\begin{aligned}
\sum_{i=1}^{4} \Phi_{4 i} \leq & \mathscr{C} \sum_{i=0}^{m-2^{E}} t_{m n\left(2^{E}+i\right) n}\left(n-2^{F}\right) \omega^{p}\left(f, 2^{-F}\right) \\
& +\mathscr{C} \sum_{i=0}^{m-2^{E}} t_{m n\left(2^{E}+i\right) n}\left(n-2^{F}\right) \omega^{p}\left(f, 2^{-F}\right) \\
& +\mathscr{C}\left(m-2^{E}\right)\left(n-2^{F}\right) t_{m n m n} \\
& \times\left(\omega^{p}\left(f, 2^{-E}\right)+\omega^{p}\left(f, 2^{-F}\right)\right),
\end{aligned}
$$

while we give a simpler form in Theorem 3 of the present paper.

Proof of Theorem 4. Since $\left\{t_{m n u v}\right\} \in \mathrm{DHBVS}$, and $m n t_{m n m n}=$ $\mathcal{O}(1)$, in the case of DHBVS, it is easy to calculate that

$$
\left\|T_{m n}-f\right\|_{p}= \begin{cases}\mathcal{O}\left(2^{-E \alpha}+2^{-F \alpha}\right), & 0<\alpha<1, \\ \mathcal{O}\left(E 2^{-E}+F 2^{-F}\right), & \alpha=1, \\ \mathcal{O}\left(2^{-E}+2^{-F}\right), & \alpha>1 .\end{cases}
$$

Note that $m \sim 2_{E}$ and $n \sim 2_{F}$; the above estimate is equivalent to

$$
\left\|T_{m n}-f\right\|_{p}= \begin{cases}\mathcal{O}\left(m^{-\alpha}+n^{-\alpha}\right), & 0<\alpha<1, \\ \mathcal{O}\left(m^{-1} \log m+n^{-1} \log n\right), & \alpha=1, \\ \mathcal{O}\left(m^{-1}+n^{-1}\right), & \alpha>1 .\end{cases}
$$

While for $\left\{t_{\text {mnuv }}\right\} \in$ DRBVS, the conclusion is obvious from Theorem 3 and the supposed $f \in \operatorname{Lip}(\alpha, p)$.

Comparing Theorems 3 and 4 with Theorems A, B, and $\mathrm{C}$, we find that the former are the generalizations of the latter, from the sense of monotonicity on one hand. On the other hand, $T$-transformation is the generalization of some means of series, such as Nörlund means, Cesàro means, and Riesz means. In the next section, we give applications of our results to some summability methods.

\section{Applications to Nörlund Means and Weighted Means}

Corollary 5. Let $T$ be the matrix defined by (11). Suppose that $\left\{p_{j k}\right\} \in D H B V S$, for $f \in L_{p}\left(I^{2}\right)$. Then, one has

$$
\begin{aligned}
P_{m n} \| & T_{m n}-f \|_{p} \\
& \leq \mathscr{C} \sum_{k=0}^{E-1} \sum_{l=0}^{F-1} 2^{k+l} p_{m-2^{k}, n-2^{l}}\left(\omega^{p}\left(f, 2^{-k}\right)+\omega^{p}\left(f, 2^{-l}\right)\right) .
\end{aligned}
$$


For $\left\{p_{j k}\right\} \in D R B V S$, one has

$$
\begin{aligned}
P_{m n}\left\|T_{m n}-f\right\|_{p} & \\
\leq & \mathscr{C} \sum_{k=0}^{E-1} \sum_{l=0}^{F-1} 2^{k+l} p_{m-2^{k+1}+1, n-2^{l+1}+1} \\
& \times\left(\omega^{p}\left(f, 2^{-k}\right)+\omega^{p}\left(f, 2^{-l}\right)\right) \\
+ & \mathscr{C} \sum_{k=0}^{E-1} 2^{k}\left(n-2^{F}\right) p_{m-2^{k+1}+1,0} \\
& \times\left(\omega^{p}\left(f, 2^{-k}\right)+\omega^{p}\left(f, 2^{-F}\right)\right) \\
+ & \mathscr{C} \sum_{l=0}^{F-1} 2^{l}\left(m-2^{E}\right) p_{0, n-2^{l+1}+1} \\
& \times\left(\omega^{p}\left(f, 2^{-E}\right)+\omega^{p}\left(f, 2^{-l}\right)\right) \\
+ & \mathscr{C}\left(m-2^{E}\right)\left(n-2^{F}\right) p_{00} \\
& \times\left(\omega^{p}\left(f, 2^{-E}\right)+\omega^{p}\left(f, 2^{-F}\right)\right) .
\end{aligned}
$$

Proof. Note that in the case when $\left\{p_{j k}\right\} \in$ DHBVS, we have $\left\{t_{m n j k}\right\} \in$ DRBVS. By the first part of Theorem 3, it is easy to deduce that

$$
\begin{aligned}
P_{m n} \| & T_{m n}-f \|_{p} \\
& \leq \mathscr{C} \sum_{k=0}^{E-1} \sum_{l=0}^{F-1} 2^{k+l} p_{m-2^{k}, n-2^{l}}\left(\omega^{p}\left(f, 2^{-k}\right)+\omega^{p}\left(f, 2^{-l}\right)\right) .
\end{aligned}
$$

When $\left\{p_{j k}\right\} \in$ DRBVS (corresponding to the case $\left.\left\{t_{m n j k}\right\} \in \mathrm{DHBV}\right)$, we get the conclusion from the second part of Theorem 3 immediately.

Remark $D$. When $\left\{p_{j k}\right\}$ is nondecreasing and $\Delta_{11} p_{j k}$ is of fixed sign, it is obvious that $\left\{p_{j k}\right\} \in$ DHBVS. Therefore, Theorem 3 of [13] can be deduced directly from the first inequality of Corollary 5. Furthermore, we exclude some preconditions in this case. For the nonincreasing case of Theorem in [13], as we figure out in Remark C, the second inequality of Corollary 5 can be seen as the generalization of this case as long as it takes the more complicated form of $\Phi_{4 i}$, $i=1,2,3,4$.

The following is a Corollary of Theorem 4, for the case of Nörlund means. Since the nondecreasing of $\left\{p_{j k}\right\}$ and fixed sign of $\Delta_{11} p_{j k}$ imply that $\left\{p_{j k}\right\} \in$ DHBVS, it is the generalization of Theorem $\mathrm{C}$.

Corollary 6. Let $T$ be the matrix defined by (11). Suppose that $\left\{p_{j k}\right\} \in D H B V S$ satisfies

$$
\frac{(m+1)(n+1) p_{m n}}{P_{m n}}=\mathcal{O}(1) .
$$

Then for $f \in \operatorname{Lip}(\alpha, p)$, one has

$$
\left\|T_{m n}-f\right\|_{p}= \begin{cases}\mathcal{O}\left(m^{-\alpha}+n^{-\alpha}\right), & 0<\alpha<1, \\ \mathcal{O}\left(m^{-1} \log m+n^{-1} \log n\right), & \alpha=1, \\ \mathcal{O}\left(m^{-1}+n^{-1}\right), & \alpha>1 .\end{cases}
$$

As far as the weighted means is concerned, we have the following corollary which generalizes Theorems A and B.

Corollary 7. Let $T$ be the matrix defined by (14) and $f \in$ $L_{p}\left(I^{2}\right)$. Suppose that $\left\{p_{j k}\right\} \in D H B V S$. Then, one has (101). For $\left\{p_{j k}\right\} \in D H B V S$, one has (100).

Suppose that $f \in \operatorname{Lip}(\alpha, p),\left\{p_{j k}\right\} \in D H B V S$ satisfies

$$
\frac{(m+1)(n+1) p_{m n}}{P_{m n}}=\mathcal{O}(1) .
$$

Then, one has

$$
\left\|T_{m n}-f\right\|_{p}= \begin{cases}\mathcal{O}\left(m^{-\alpha}+n^{-\alpha}\right), & 0<\alpha<1, \\ \mathcal{O}\left(m^{-1} \log m+n^{-1} \log n\right), & \alpha=1, \\ \mathcal{O}\left(m^{-1}+n^{-1}\right), & \alpha>1 .\end{cases}
$$

\section{Conflict of Interests}

The authors declare that there is no conflict of interests regarding the publication of this paper.

\section{References}

[1] A. Paley, "A remarkable series of orthogonal functions," Proceedings of the London Mathematical Society, vol. 34, no. 1, pp. 241$279,1932$.

[2] M. S. Corrington, "Solution of differential and integral equations with Walsh functions," IEEE Transactions on Circuit Theory, vol. 20, no. 5, pp. 470-476, 1973.

[3] R. R. Coifman and M. V. Wickerhauser, "Entropy-based algorithms for best basis selection," IEEE Transactions on Information Theory, vol. 38, no. 2, pp. 713-718, 1992.

[4] P. N. Paraskevopoulos, "Chebyshev series approach to system identification, analysis and optimal control," Journal of the Franklin Institute, vol. 316, no. 2, pp. 135-157, 1983.

[5] G. B. Mahapatra, "Solution of optimal control problem of linear diffusion equations via Walsh functions," IEEE Transactions on Automatic Control, vol. 25, no. 2, pp. 319-321, 1980.

[6] F. Schipp, W. R. Wade, P. Simon, and J. Pal, Walsh Series: An Introduction to Dyadic Harmonic Analysis, Adam Hilger, Bristol, UK, 1990.

[7] B. I. Golubov, A. V. Efimov, and V. A. Skvortsov, Ryady i Preobrazovaniya Uolsha. Teoriya i Primeneniya, Nauka, Moscow, Russia, 1987, English Translation: Walsh Series and Transforms. The Theory and Applications, Kluwer Academic, Dordrecht, The Netherlands, 1991.

[8] U. Goginava, "Approximation properties of $(C, \alpha)$ means of double Walsh-Fourier series," Analysis in Theory and Applications, vol. 20, no. 1, pp. 77-98, 2004. 
[9] S. Yano, "On Walsh-Fourier series," The Tohoku Mathematical Journal, vol. 3, pp. 223-242, 1951.

[10] E. Savaş and B. E. Rhoades, "Necessary conditions for inclusion relations for double absolute summability," Proceedings of the Estonian Academy of Sciences, vol. 60, no. 3, pp. 158-164, 2011.

[11] F. Móricz and A. H. Siddiqi, "Approximation by Nörlund means of Walsh-Fourier series," Journal of Approximation Theory, vol. 70, no. 3, pp. 375-389, 1992.

[12] F. Móricz and B. E. Rhoades, "Approximation by weighted means of Walsh-Fourier series," International Journal of Mathematics \& Mathematical Sciences, vol. 19, no. 1, pp. 1-8, 1996.

[13] K. Nagy, "Approximation by Nörlund means of double WalshFourier series for Lipschitz functions," Mathematical Inequalities \& Applications, vol. 15, no. 2, pp. 301-322, 2012.

[14] L. Leindler, "On the degree of approximation of continuous functions," Acta Mathematica Hungarica, vol. 104, no. 1-2, pp. 105-113, 2004. 


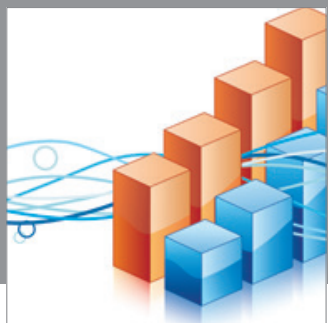

Advances in

Operations Research

mansans

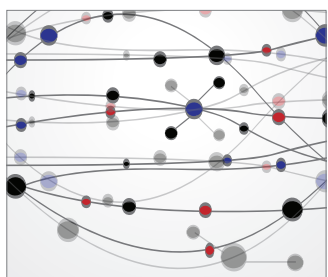

The Scientific World Journal
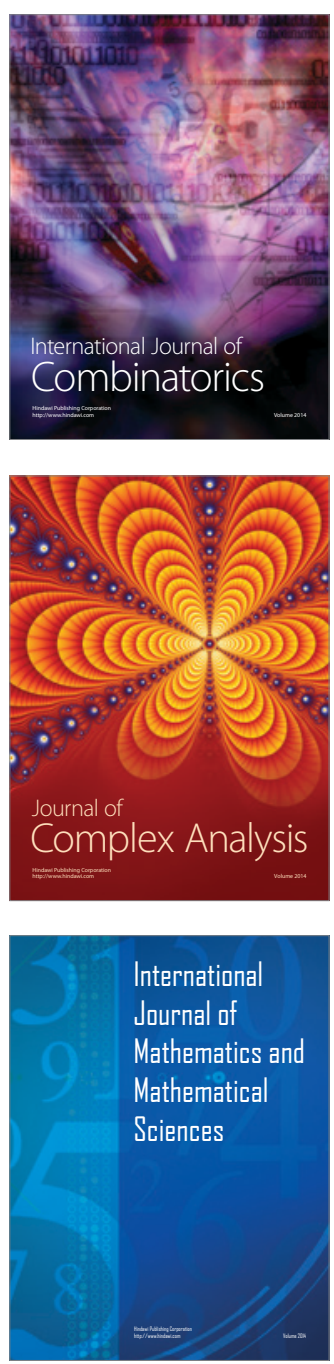
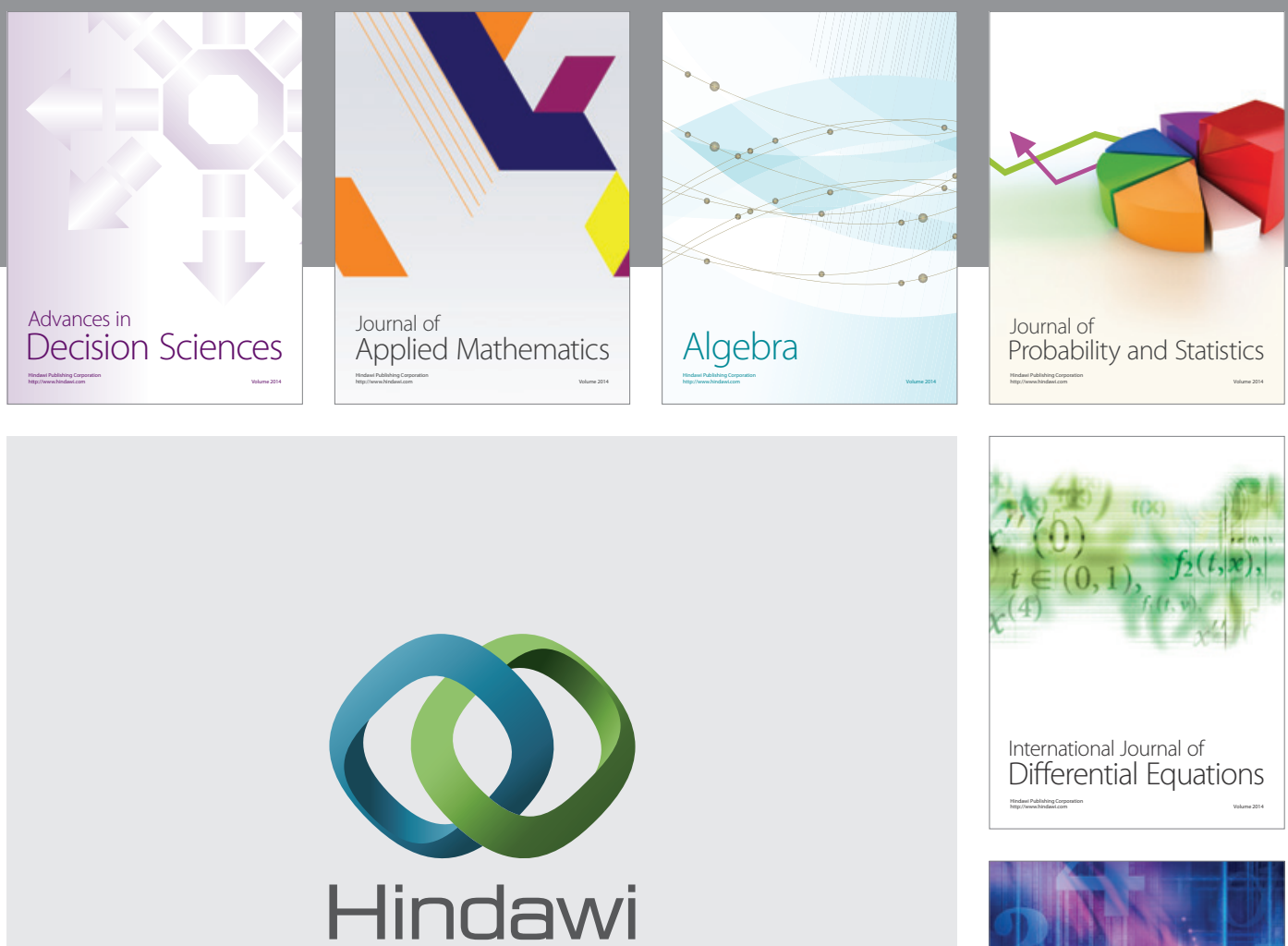

Submit your manuscripts at http://www.hindawi.com
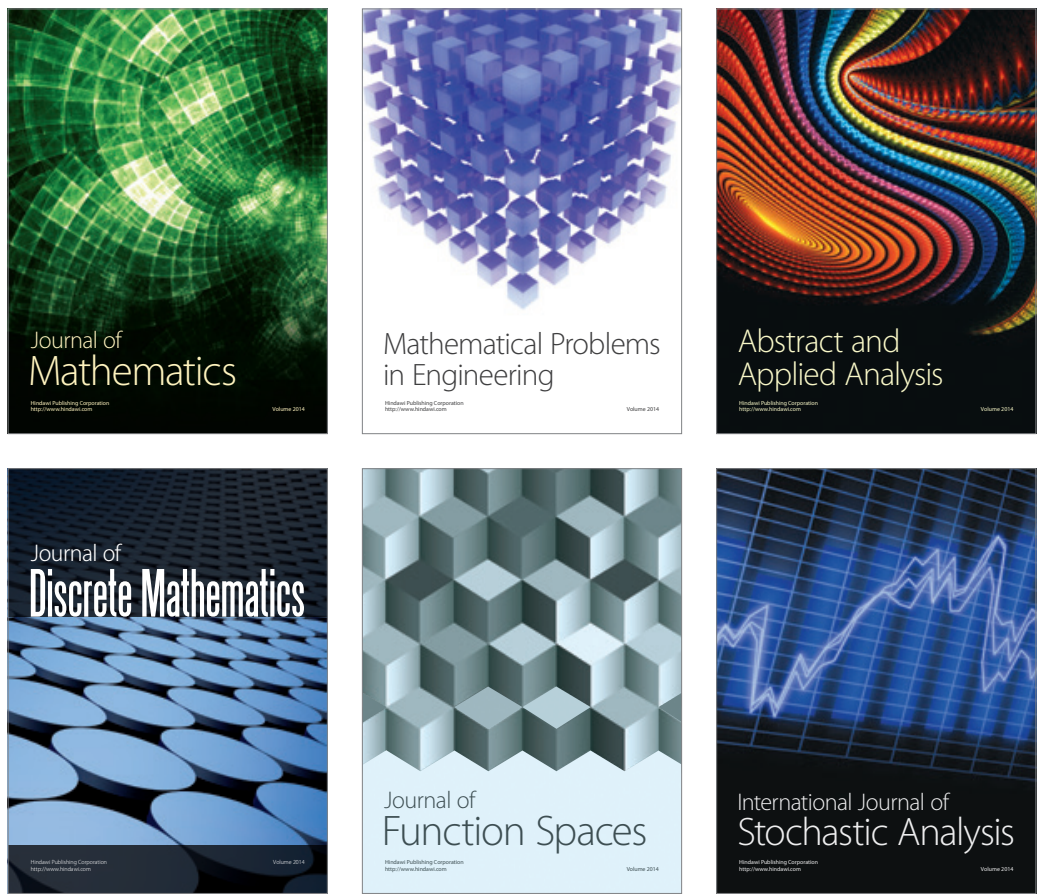

Journal of

Function Spaces

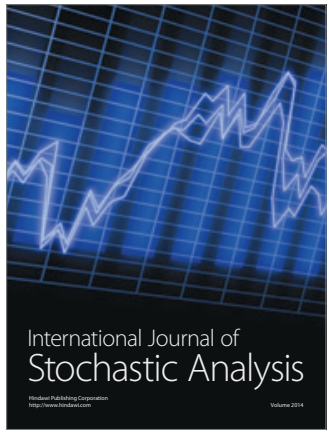

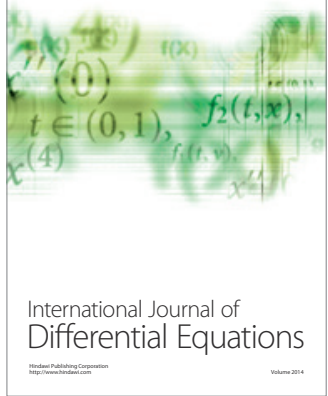
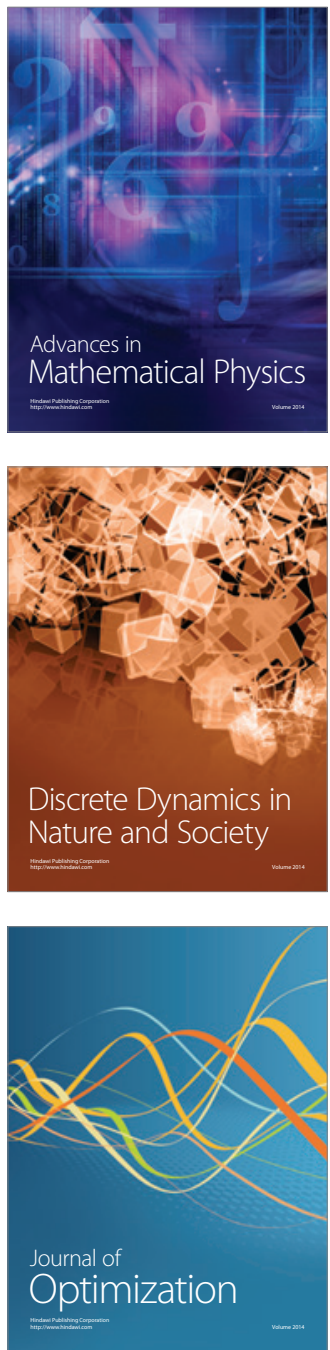